\title{
Pseudoaneurysm of Ascending Aorta with Sternal Erosion after Double Valve Replacement-A Case Report
}

\author{
Ravi Kumar Kathi ${ }^{1}$ Amaresh Rao Malempati ${ }^{1}$ Goutham Kollapalli ${ }^{1}$ Chaitra Krishna Batt ${ }^{1}$ \\ Sayyad Sohail Tarekh ${ }^{1}$ \\ ${ }^{1}$ Nizam's Institute of Medical Sciences, Panjagutta, Hyderabad, \\ Telangana, India

\begin{abstract}
Address for correspondence Amaresh Rao Malempati, MS, MMch, Additional Professor CT Surgery, Nizam's Institute of Medical Sciences, Panjagutta, Hyderabad 500082, Telangana, India (e-mail: raoamareshm@yahoo.co.in).
\end{abstract}

Ind J Car Dis Wom 2019;4:92-94

\begin{abstract}
Pseudoaneurysm of ascending aorta is a rare complication after aortic surgery. Predisposing factors can be infection, chronic hypertension, connective tissue disorders, or dissection. Chest pain, sternal erosion, dysphagia, or stridor can be the modes of presentation. It can also present as a pulsatile mass. Redo sternotomy in a case

Keywords

- pseudoaneurysm

- cardiopulmonary bypass

- deep hypothermic circulatory arrest of pseudoaneurysm of aorta can cause fatal hemorrhage or air embolism. In such a scenario, femorofemoral bypass and hypothermic circulatory arrest help to simplify the approach to the pseudoaneurysm. The authors present a case of a 23-year-old female with pseudoaneurysm of the ascending aorta causing sternal erosion. Ascending aortic repair was done using Dacron patch with femorofemoral bypass and hypothermic circulatory arrest. Sternum was repaired using pectoralis major muscle flap.
\end{abstract}

\section{Introduction}

Thoracic aortic pseudoaneurysm is an uncommon complication of cardiac or aortic surgery. Its incidence is $<0.5 \%{ }^{1}$ The cause is disruption of the intima and media of the aorta with the resultant swelling being contained by the adventitia and the surrounding mediastinal structures. Other factors can be either trauma or infection. ${ }^{2}$ The major risk factor for ascending aortic pseudoaneurysm is leak from aortic cannulation site. ${ }^{3}$

Pseudoaneurysms can also occur at previous venting, aortotomy, anastomotic site or proximal vein graft sites. The various mechanisms implicated are anastomotic technique, infection, and intrinsic aortic wall disease. The lesion can be detected initially by a chest X-ray (posteroanterior view), which will show widening of the mediastinum. The diagnostic test is computed tomographic aortogram (CT aortogram). ${ }^{4}$ Repair of such a fragile lesion is a surgical challenge.

\section{Case Presentation}

A 23-year-old female patient presented with complaints of fever on and off for 6 months and pulsatile swelling in front of the chest for 1 month. One year back, she was operated for rheumatic valvular heart disease of severe mitral and aortic stenosis with mitral valve replacement of $25 \mathrm{~mm}$ and aortic valve replacement of $19-\mathrm{mm}$ Chitra valves. She was not a known hypertensive or diabetic. On examination, the patient was febrile with a temperature of $101^{\circ} \mathrm{F}$. Pulsatile mass of $8 \times 7 \mathrm{~cm}$ was noted in front of sternum ( - Fig. 1). On chest X-ray, mediastinal widening was present ( Fig. 2). Two-dimensional (2D) echo showed that both prosthetic valve leaflets were moving well, no increased gradients across valves or paravalvular leak were noted, and biventricular function was good. On a CT aortogram, pseudoaneurysm of size $8 \times 7 \mathrm{~cm}$ size was noted (-Figs. 3 and $\mathbf{4}$ ).

We operated on the patient with a femorofemoral bypass with deep hypothermic circulatory arrest for 20 minutes. After circulatory arrest was achieved on femorofemoral bypass, midline sternotomy was done. Intraoperatively, there were dense adhesions present in the mediastinum (-Fig. 5). We removed all blood clots and released adhesions. After clearing the surgical field, we noted a defect of size $3 \times 2 \mathrm{~cm}$ at the aortotomy site on the right side ( $\mathbf{- F i g}$. $\mathbf{6}$ ), closed the defect with Dacron patch, and reinforced with pledgeted sutures ( - Fig. 7 ). After that cardiopulmonary bypass reinitiated, the patient was 


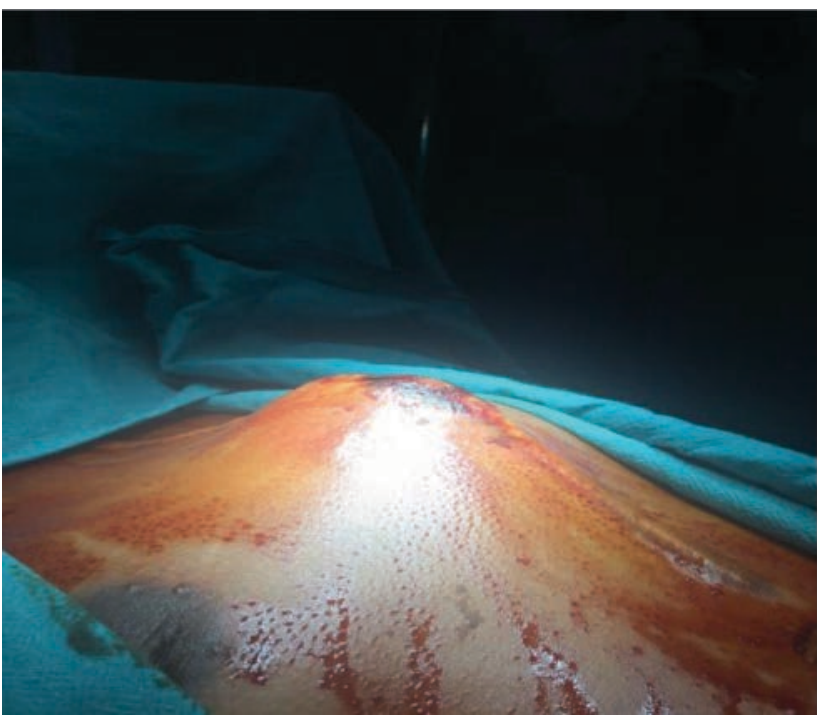

Fig. 1 Pseudoaneurysm eroding the sternum with impending rupture.

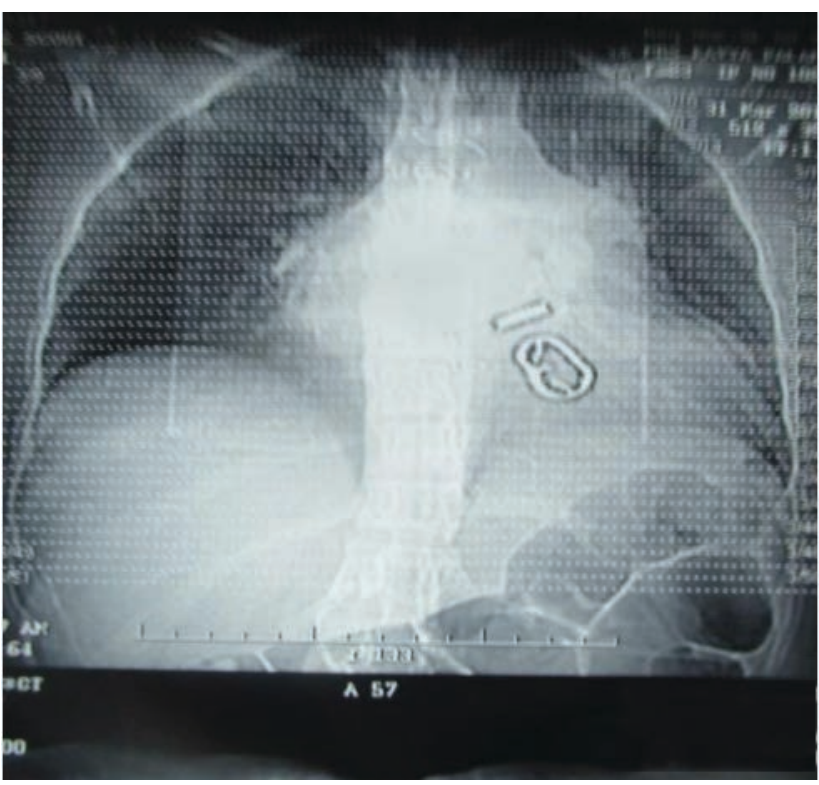

Fig. 2 Mediastinal widening on chest X-ray.

rewarmed to $36^{\circ} \mathrm{C}$ and came off bypass in normal sinus rhythm. We temporarily closed the skin, and after 24 hours, sternal reconstruction was done with pectoralis muscle flap ( - Fig. 8). Postoperatively, the patient was kept on broad-spectrum antibiotics and was discharged on 15th postoperative day. On follow-up, the patient was doing well till then.

\section{Discussion}

Morbidity and mortality of pseudoaneurysm of the ascending aorta is between 29 and $46 \%{ }^{5}$ This is due to the fatal hemorrhage from rupture of the lesion. Therefore, a pseudoaneurysm should be operated upon soon after diagnosis. When such a lesion is located anteriorly, is large and eroding into the sternum, risk of massive hemorrhage during sternotomy or surgery is very high. ${ }^{6}$ So it is important to be careful during redo sternotomy. Another important aspect is preservation of cerebral perfusion. ${ }^{6}$ Authors have described various methods of initiating cardiopulmonary bypass prior

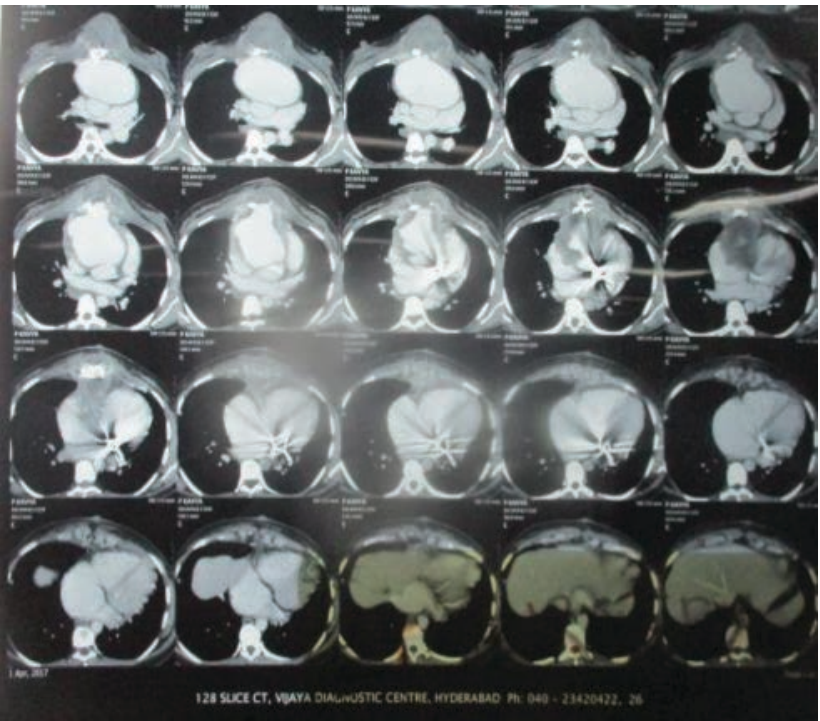

Fig. 3 CT scan showing pseudoaneurysm eroding the sternum.

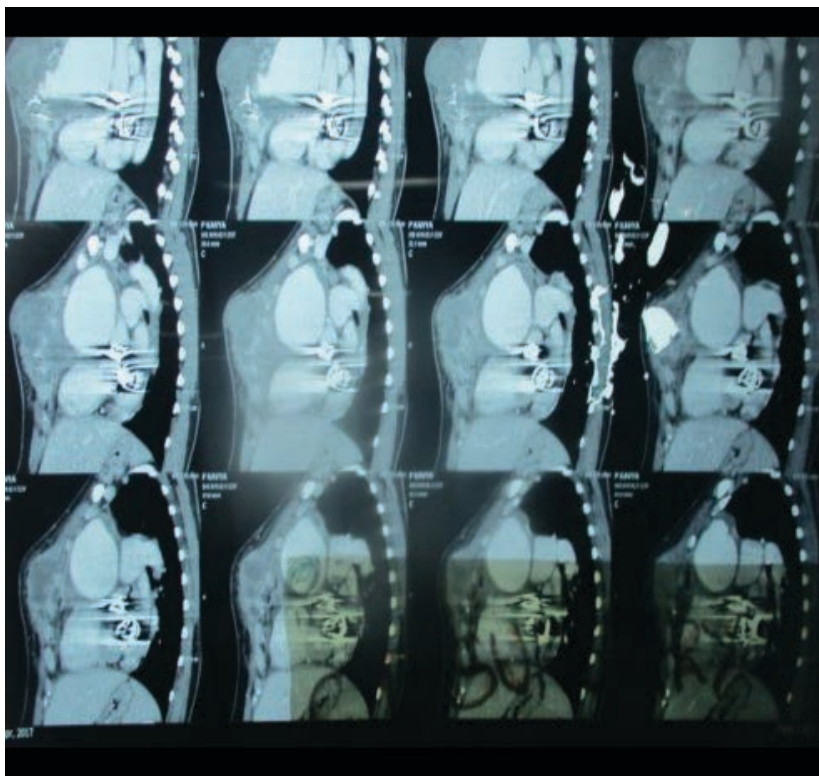

Fig. 4 Sagittal section of CT scan showing sternal erosion.

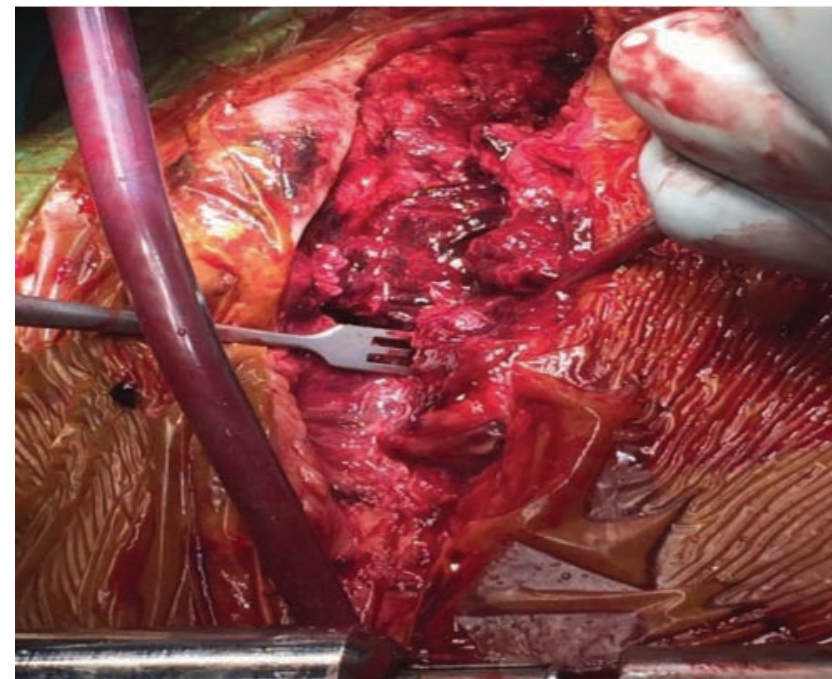

Fig. 5 Intraoperative picture showing instrument at edge of eroded sternum after clot evacuation. 


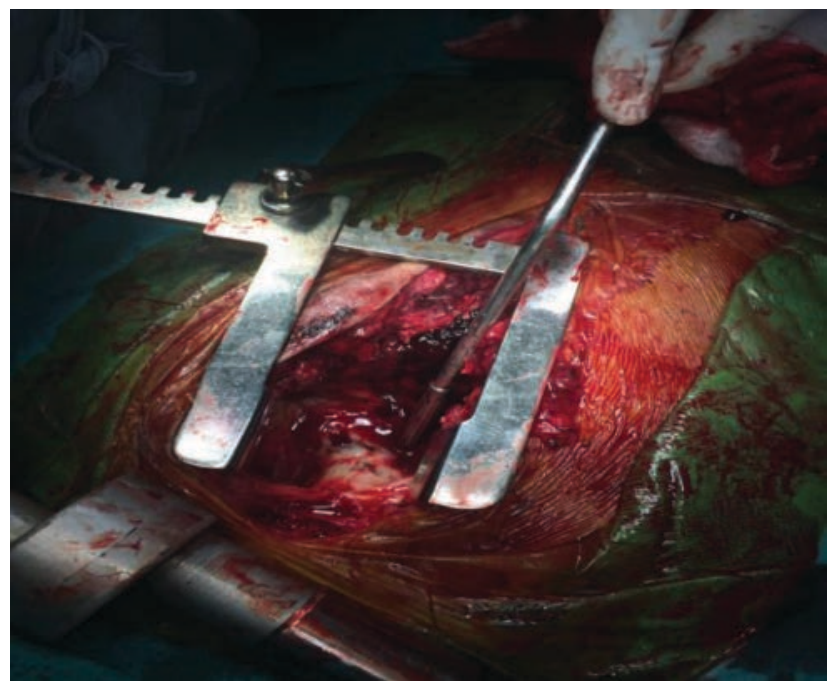

Fig. 6 Instrument pointing to rent in ascending aorta.

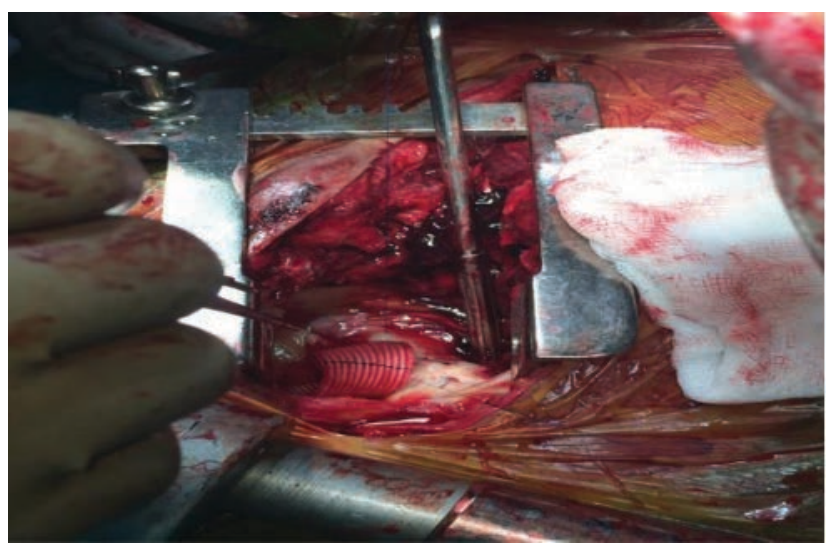

Fig. 7 Closing of rent with Dacron patch.

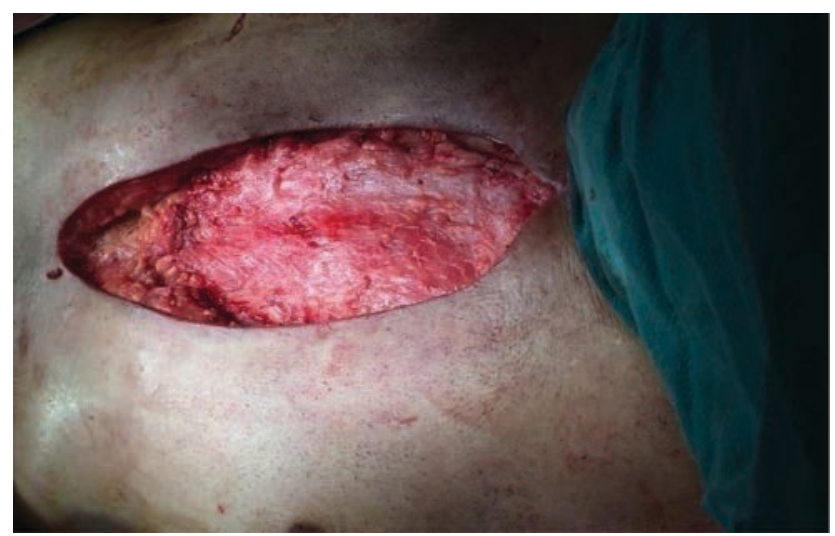

Fig. 8 Sternal reconstruction with pectoralis major muscle flap.

to redo sternotomy. ${ }^{1,6,7}$ The best approach depends on the site and the size of the pseudoaneurysm. Femoral or axillary artery cannulation has been suggested as the most feasible.

Mohammadi et $\mathrm{a}^{8}$ used bilateral carotid artery cannulation and femorofemoral bypass in nine patients. Bachet et al, ${ }^{7}$ in 2007, directly cannulated both carotids in five patients. They argued that it preserved cerebral blood flow and also maintained systemic flow. However, subjects in both these studies experienced neurological sequelae. Carotid artery cannulation has not been widely used due to the risk of stroke.

Alternative novel approaches such as use of atrial septal defect occluder device for closure of the pseudoaneurysm might be tried in selected patients. ${ }^{9}$

Hypothermic circulatory arrest decreases the intravascular volume and provides cerebral protection. Thereby, it gives the surgeon some time to dissect, define, and repair the lesion. It also prevents sudden, extreme blood loss. ${ }^{10}$

\section{Conclusion}

Repair of a large pseudoaneurysm of the ascending aorta via redo median sternotomy entails high risk of fatal hemorrhage. Femorofemoral bypass and moderate hypothermia with transient circulatory arrest help safe conduct of surgery.

\section{Conflicts of Interest}

None.

\section{References}

1 Katsumata T, Moorjani N, Vaccari G, Westaby S. Mediastinal false aneurysm after thoracic aortic surgery. Ann Thorac Surg 2000;70(2):547-552

2 Aebert H, Birnbaum DE. Tuberculous pseudoaneurysms of the aortic arch. J Thorac Cardiovasc Surg 2003;125(2):411-412

3 Sullivan KL, Steiner RM, Smullens SN, Griska L, Meister SG. Pseudoaneurysm of the ascending aorta following cardiac surgery. Chest 1988;93(1):138-143

4 Moore EH, Farmer DW, Geller SC, Golden JA, Gamsu G. Computed tomography in the diagnosis of iatrogenic false aneurysms of the ascending aorta. AJR 1984;142:117-118

5 Attia R, Venugopal P, Whitaker D, Young C. Management of a pulsatile mass coming through the sternum. Pseudoaneurysm of ascending aorta 35 years after repair of tetralogy of Fallot. Interact Cardiovasc Thorac Surg 2010;10(5):820-822

6 Garisto JD, Medina A, Williams DB, Carrillo RG. Surgical management of a giant ascending aortic pseudoaneurysm. Tex Heart Inst J 2010;37(6):710-713

7 Bachet J, Pirotte M, Laborde F, Guilmet D. Reoperation for giant false aneurysm of the thoracic aorta: how to reenter the chest? Ann Thorac Surg 2007;83(5):1610-1614

8 Mohammadi S, Bonnet N, Leprince P, et al. Reoperation for false aneurysm of the ascending aorta after its prosthetic replacement: surgical strategy. Ann Thorac Surg 2005;79(1):147-152, discussion 152

9 Kassaian SE, Forouzannia SK, Hosseinsabet A, Davarpasand T. Novel treatment approach to ascending aorta pseudoaneurysms: a case report. J Tehran Heart Cent 2017;12(3):134-137

10 Darmoch F, Moussa Pacha H, Pagadala P, Alraies MC. Pseudoaneurysm of the ascending thoracic aorta years after aortic valve replacement. Cardiovasc Revasc Med 2018;19(2):196-98 